\title{
Coherent excitonic effects in quantum wells
}

\author{
J. SHAH and D.S. KIM
}

AT\&T Bell Laboratories, Holmdel, NJ 07733, U.S.A.

\begin{abstract}
Coherent transient spectroscopy of semiconductor nanostructures has become an active field of research in recent years. Most such experiments are analyzed in terms of a simple two-level model in which the effects of interaction and induced fields are ignored. We discuss two very different cases where inclusion of these generally-neglected effects leads to large and important modification in the temporal response of the system. In the first case, interactions between excitons strongly modify the temporal response of four-wave-mixing (FWM) signals from quantum wells. This is the temporal analog of well-known effects in the spectral domain, such as bleaching of the exciton absorption and bandgap renormalization. In the second case, the shape of a low-intensity pulse is severely distorted after transmission through a typical quantum well sample. Interferometric measurements show that reradiation of induced polarization is responsible for the distortion. The distortion depends strongly on the dephasing time and has important implications for all time-resolved measurements.
\end{abstract}

\section{INTRODUCTION}

Coherent spectroscopy of excitons in semiconductor quantum wells is an active field of research. Early studies included investigations of exciton dephasing $[1,2]$ while recent results include observation of quantum beats [3-5], coherent oscillations of wavepackets in coupled quantum wells [6] and superlattices [7], and coherent submillimeter-wave radiation from oscillating charge packets [8]. Most of the results of coherent spectroscopy are analyzed in terms of a simple two-level model which ignores (1) exciton-exciton interaction, and (2) reradiation by the induced polarization. The first effect is independent of the density and sample thickness, whereas the second effect is important for all experiments except in optically-thin samples. We review in this paper recent results [9-11] which show that these effects make extremely important contributions to the coherent response of excitons.

\section{BASIC CONCEPTS}

The coherent response of excitons is analyzed using the Semiconductor Bloch Equations (SBE, which include the interactions between excitons [12]), coupled with the Maxwell equations. The SBE equations for an interacting two-level system can be written in a simplified form as:

$$
\begin{gathered}
\dot{\psi}+\left(1 / T_{2}+i \omega_{0}\right) \psi-i(1-2 n)(\mu E+V \psi) / \hbar=0 \\
\dot{n}+n / T_{1}+\frac{2}{\hbar} \operatorname{Im}\left((\mu E+V \psi) \psi^{*}\right)=0
\end{gathered}
$$


Here $T_{2}$ is the exciton dephasing time, $T_{1}$ is the exciton recombination time, $\hbar \omega_{0}$ is the exciton transition energy, $E$ is the electric field amplitude, $\mu$ is the dipole matrix element of the transition, and $n$ and $\psi$ are the diagonal and off-diagonal components of the density matrix representing the exciton population and coherent polarization respectively. In this simplified SBE, $V$ is an average interaction potential between excitons. In the absence of interactions, $V=0$ and the SBE reduce to the optical Bloch equations for non-interacting (independent) two-level system.

The electric field amplitude in these equations is in general not the electric field amplitude of the incident field, but must be determined by coupling these equations to the Maxwell's equation which relates the electric field to the polarization $P=N \mu \psi$ :

$$
\left(\frac{\partial}{\partial z}+\frac{1}{v} \frac{\partial}{\partial t}\right) E=i 2 \pi \omega P / c
$$

The solution of these coupled equations is quite complicated in general. However, in order to obtain physical insights into the nonlinear behavior, one often makes many simplifications. One of the most commonly made assumptions involves ignoring the interaction between excitons $(V=0)$. Under this approximation, the Rabi frequency which determines the interaction Hamiltonian depends only on the electric field amplitude of the incident electromagnetic field. The second common assumption is to assume that the sample is optically thin so that the electric field amplitude is given by the amplitude of the incident field. This second assumption eliminates the need to consider equation (3). These assumptions lead to an enormous simplification of the equations, and simple predictions can be made for the temporal response of the FWM signals for delta-function pulses, for the case of homogeneous and inhomogeneous broadening [13]. We consider the predictions of this simple model in this section. Sections 3 and 4 will present experimental data which show that these assumptions are not justified for excitons in quantum wells; $i$. e. exciton-exciton interaction and propagation effects lead to strong modifications of the nonlinear response of excitons in quantum wells.

Fig. 1 (right) a schematic diagram for timeintegrated (TI) and time-resolved (TR) four-wave-mixing (FWM) measurements. The experiment is performed with two trains of ultrashort pulses delayed by a time delay $T$. The first pulse along $\bar{k}_{1}$ produces a polarization in the semiconductor; the grating produced by the interference between the second pulse along $\bar{k}_{2}$ and this polarization diffracts a part of the second pulse along the direction $2 \bar{k}_{2}-$ $\bar{k}_{1}$. In TI-FWM, one measures the total diffracted energy as a function of T, whereas in TR-FWM one measures the temporal shape of the diffracted pulse at various $T$. The

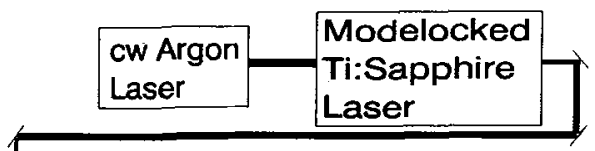
two kinds of experiments provide different information.

The dot-dashed curve in Fig. 2 (right) shows the TR-FWM signal as a function of time $\mathrm{t}$ (normalized to $T_{2}$ ) for delta-function pulses for the case of homogeneouslybroadened two-level system in which interaction between excitons is neglected. TRFWM rises instantaneously at $t=0$ and then decays exponentially with decay constant $\tau_{\text {decay }}$ given by $T_{2} / 2$.

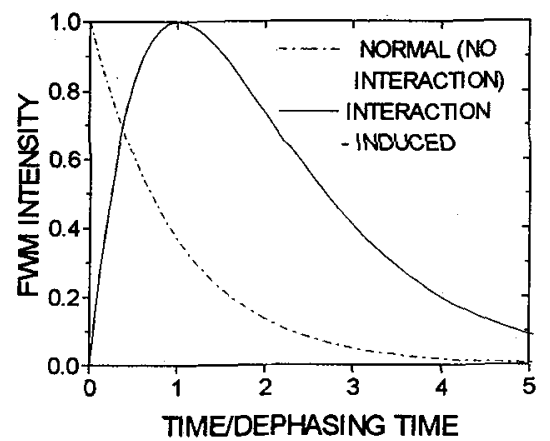




\section{EXCITON-EXCITON INTERACTION}

\subsection{Basic Concepts}

The predictions of this simple model are modified in two important ways if one considers the interaction between excitons. As can be seen from Eq. 1 and Eq. 2 [12], the influence of excitonic interaction is to (1) renormalize the energy separation between the two levels, and (2) renormalize the Rabi frequency which characterized the interaction Hamiltonian. The remormalization of the Rabi frequency leads to the scattering of polarization in addition to the usual scattering of electric field. Under short-pulse excitation, the polarization field can persist much after the passage of the laser pulse. The diffraction of polarization then leads to a signal at negative time delays, $\mathrm{T}<0$. For $\mathrm{T}>0$, the interaction leads to an additional contribution to the diffracted signal which increases slowly after the arrival of the second pulse and peaks at time $t$ approximately equal to $T_{2}$, where time $t$ is measured from the second pulse. This is interaction-induced signal is illustrated the (solid curve) in Fig. 2. Although the normal and interaction-induced components of the TR-FWM signal are shown normalized in Fig. 2, their relative importance depends on the strength of the interaction and the dephasing time. Experiments provide information about the relative strengths.

\subsection{FWM Signal at Negative Time Delays}

TI-FWM signals from a high quality GaAs quantum well sample with $175 \AA$ well-width was investigated by Leo et al. [9]. The exciton linewidth was $0.7 \mathrm{meV}$ and there was no Stokes shift between absorption and emission. TI-FWM signals from the sample for various temperatures is shown in Fig. 3 (below left). The rise and decay times of the TI-FWM signals at various temperatures are plotted in Fig. 4 (below right). At the lowest temperatures, the results clearly show a negative timedelay component to the signal with the risetime much larger than the time resolution of the system. From a simple theory it is expected that the risetime is given by $\mathrm{T}_{2} / 4$ whereas the decay time is given by $\mathrm{T}_{2} / 2$, so that the ratio is 2 . The measured ratio at low temperatures is approximately two, as expected theoretically. With increasing temperature, both the rise and the decay times decrease as the dephasing time $T_{2}$ decreases, but the ratio continues to be close to 2 until the results become limited by the time resolution ( $0.3 \mathrm{ps})$.
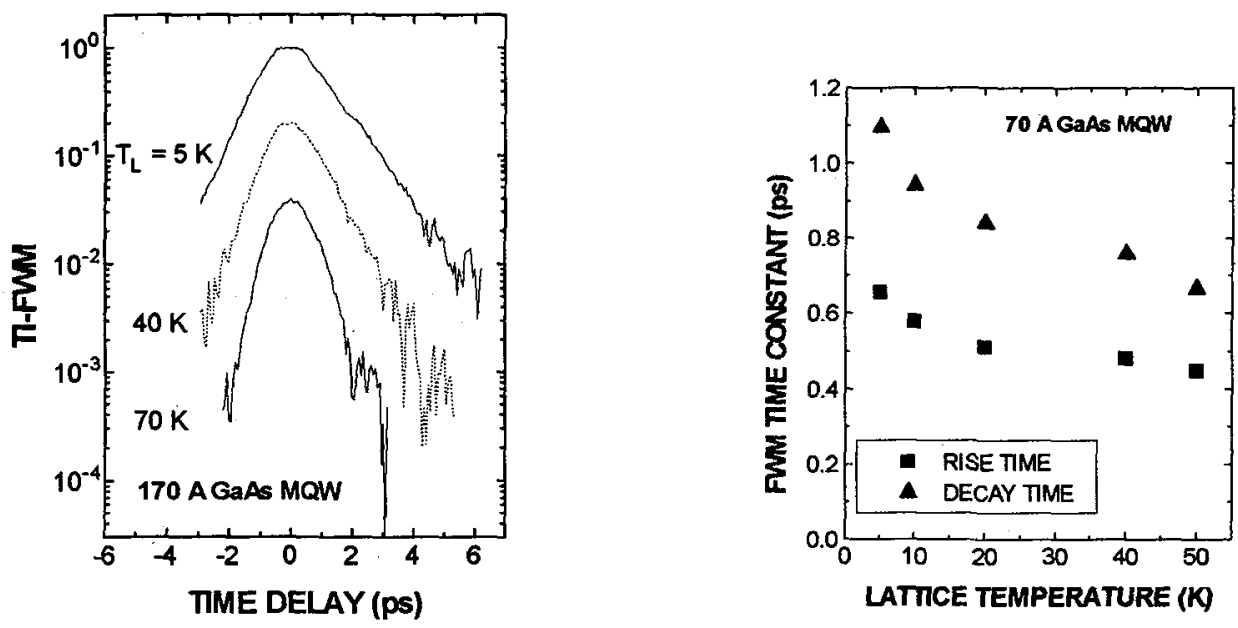

These results clearly show the influence of exciton interaction on the nonlinear response of the quantum wells. However, they do not provide any information on the relative strengths of the interaction-induced and normal signal. A more sophisticated experiment is required to obtain this information. 


\subsection{Slow Rise of FWM Signals}

The TR-FWM signals from the same sample were measured with 100 fs pulses by Kim et al. [11]. Fig. 5 (above right) shows the results for three different temperatures for $T=1$ ps. At the lowest temperature the peak of the signal occurs at approximately $1 \mathrm{ps,}$ considerably beyond the expected position of the peak for the normal signal using 100 fs pulses. Increasing the temperatures brings the peak closer to $t=0$, showing that the signal is not due to photon echo. It is known that increasing temperature reduces $T_{2}$ (see, for example Fig. 3) so the position of the peak depends on $T_{2}$. This is precisely the property expected for the interaction-induced signal, as discussed in Sec. 2.1. This signal was identified with the interaction-induced signal [11]. These results show that interactioninduced signal completely dominates the FWM experiments.

Theoretical analysis shows that the signal at $\mathrm{t}=\tau_{p}$, where $\tau_{p}$ is the pulsewidth of

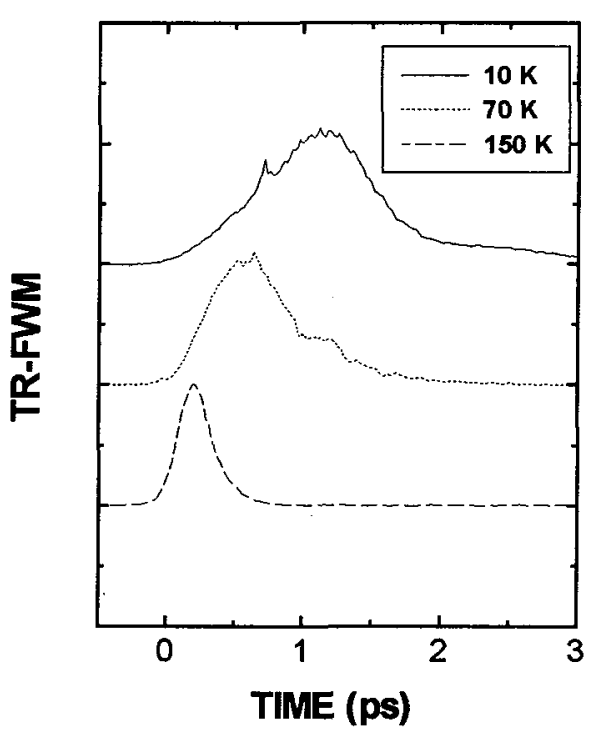
the laser, approximately corresponds to the normal signal whereas the signal at the peak corresponds to the interaction-induced signal. The ratio of the interaction-induced to the normal signal is given by $\left[V T_{2} / \hbar\right]^{2}$. It was deduced from these results [11] that the exciton interaction potential $V$ is $1-2 \mathrm{meV}$, a value that is quite reasonable.

\subsection{Summary}

These results show that excitonic interaction leads to characteristic temporal signatures in the coherent transients, and its investigation provides interesting insights into the interaction as well as the nature of nonlinear response of quantum wells.

\section{DISTORTION OF LOW-INTENSITY PULSES}

\subsection{Basic Concepts}

The renormalization of the Rabi frequency discussed in the previous section can be viewed as replacing the incident electromagnetic field with a local field that is the sum of the incident field and a quantity proportional to the polarization. Although one talks about a local field correction, this correction occurs only in the interaction term and the field amplitude occurring elsewhere in SBE is equated to the incident field amplitude. This simplifying assumption is valid only when the optical thickness of the sample is small, as we discussed in Sec. 2.

In this sub-section we consider a simple case in which the renormalizations of the Rabi frequency and the transition energy are ignored $(V=0)$, and the intensity of the incident radiation is assumed to be so low that the excited-state population produced by the laser is ignored in the analysis $(n=0)$. The electric field amplitude is not assumed to be that of the incident field but calculated from coupled equations 2 and 3 . Under these conditions, it has been shown that the transmitted pulse shows aperiodic oscillations in time [14]. For a solid, the exciton-polariton dispersion relation has also to be considered, as for the case of $\mathrm{Cu}_{2} \mathrm{O}$ [15]. The polaritons in 2D systems are more complicated than in bulk crystals, and in particular do not show the two-mode behavior of the bulk polaritons [16]. However, analysis shows that the coupled equation analysis still leads to aperiodic oscillations [17]. 
In the next sections, we present some recent experimental results on the strong distortion of lowintensity pulses propagating through a moderately thick quantum well sample. We then discuss interferometric measurements that shed light on the origin of this behavior, and finally discuss the implications of these results on pump-and-probe measurements.

\subsection{Distortion of Low-Intensity Pulses}

We discuss in this section some recent results obtained by Kim et al. [16]. Fig. 6 (right) shows the temporal shape of a lowintensity pulse after propagating through a sample with 75 periods of $84 \AA \mathrm{GaAs} / \mathrm{Al}_{0.3} \mathrm{Ga}_{0.7}$ As quantum wells at $10 \mathrm{~K}$. The incident pulse from a Ti:Sapphire laser (pulsewidth of $\approx 100 \mathrm{fs}$ ) was centered at the $\mathrm{HH}$ exciton of the sample, and produced $10^{6}$ carriers $/ \mathrm{cm}^{2}$ per well. The transmitted pulseshape is clearly very strongly distorted, with the distortion comparable to the initial transmitted peak, and shows aperiodic oscillations. The distortion in a thinner sample (total thickness of $1700 \AA$ was 2 orders of magnitude smaller and corresponds to the free induction decay (FID) of the polarization produced in the sample. However, the strong distortion in the $6300 \AA$ sample cannot be described in terms of FID and should be viewed as resulting from a superposition of

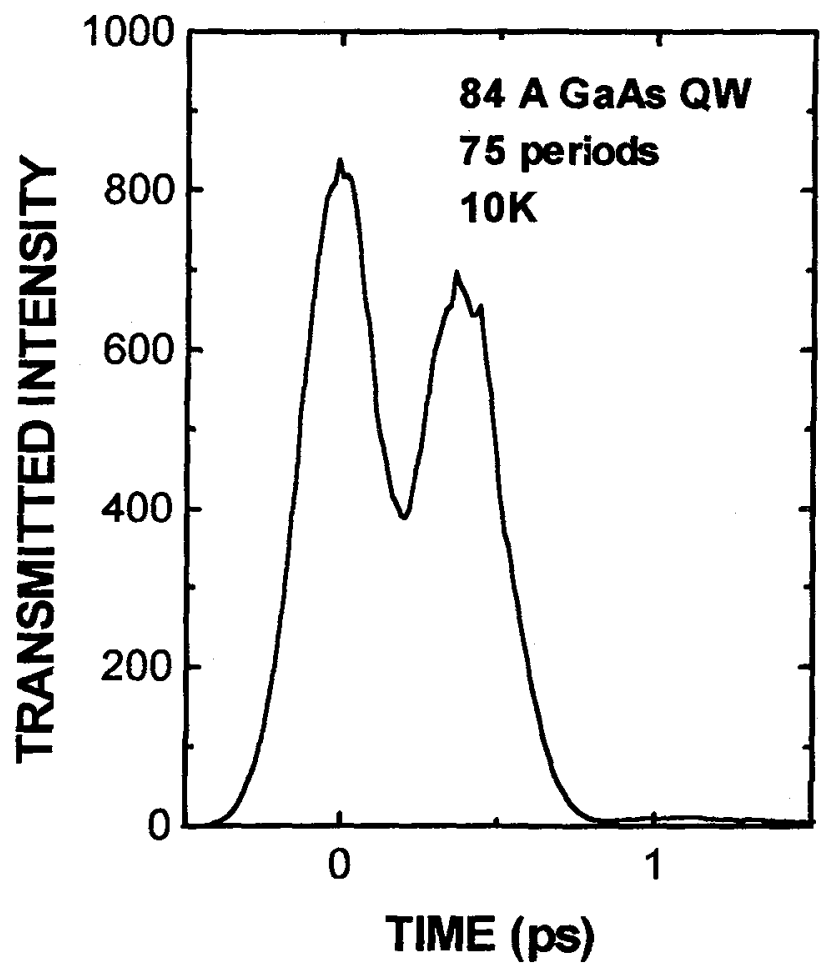
the incident field and the field reradiated by the polarization created in the quantum wells.

\subsection{Interferometric Measurements}

This point is made more clearly by the interferometric measurements performed by Kim et al. [17] using a modified Michelson interferometer. Such a measurement allows the determination of the amplitude and the phase of the total radiated field in the transmitted direction. The advantage of this modified setup is that the pulse passes through the sample only once.

Fig. 7 (top of next page, left) shows the results of such a measurement. The envelope of the total radiated field clearly shows nodes at the same position as the minima in the transmitted intensity in Fig. 6. Furthermore, an expanded view near the first node, shown in Fig. 8 (top of next page, right) clearly shows that there is a $180^{\circ}$ change of phase at the node. Thus, the initial part of the pulse, upto the first node, is primarily due to the transmitted pulse, whereas the subsequent part is due to the reradiation by the polarization created in the quantum wells. More complicated patterns of the transmitted amplitude and phase are observed when the incident laser is not centered at the $\mathrm{HH}$ exciton [18]. 

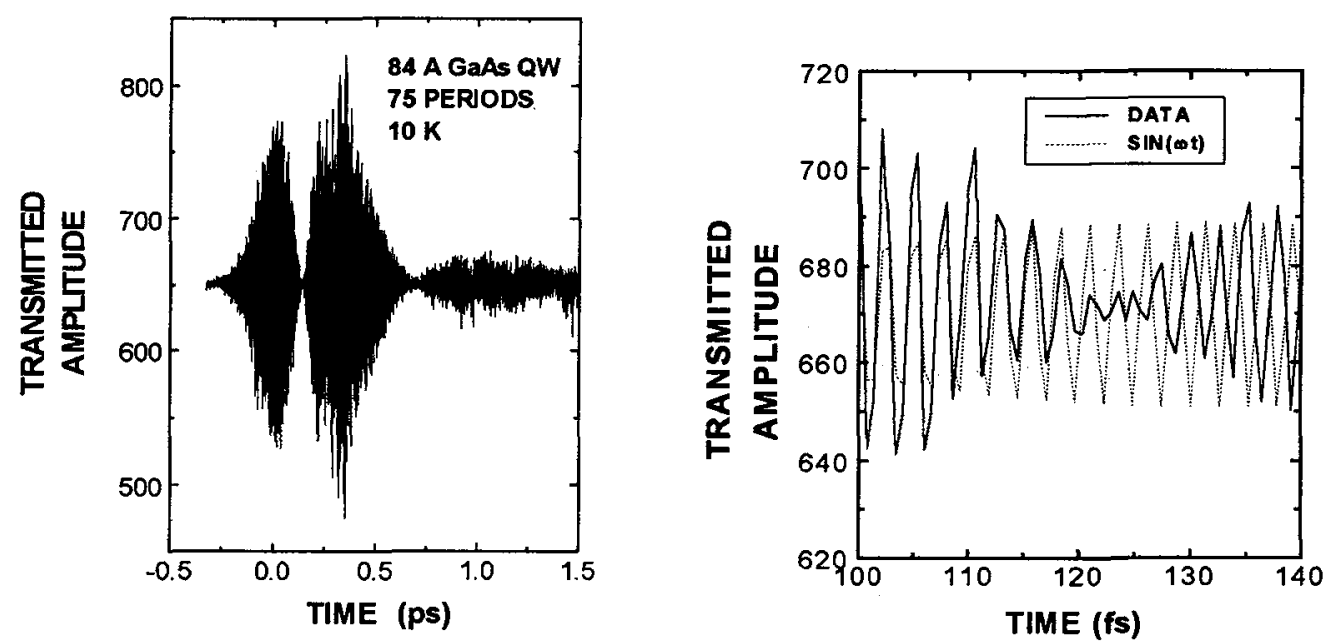

\subsection{Implications for Exciton Density}

We have numerically fitted the observed shape of the transmitted pulse solving the coupled equations (1) and (3). With these parameters, we can also solve for the density $n$ of excitons from Eq. (2). The results of such a calculation for the exciton density at the end of the sample shows an interesting non-monotonic (oscillatory) temporal behavior (Fig. 9, right). Further investigations of this behavior will be published elsewhere [18].

\subsection{Implications for Pump-probe Measurements}

As we mentioned earlier, the observed distortion for a given optical thickness of the sample is strongly influenced by the homogeneous and inhomogeneous excitonic linewidths. For a given homogeneous linewidth (or dephasing time), the distortion becomes weaker if the inhomogeneous linewidth increases, because the macroscopic polarization then decays faster. For a given inhomogeneous width, the distortion becomes weaker as the homogeneous linewidth increases or the dephasing time decreases. Thus, if the probe pulse being measured is preceded by a strong pump pulse which produces a moderately strong excitation of the sample and reduced the dephasing time of the excitation, then the distortion becomes smaller. This is illustrated in Fig. 10 (right). The transmitted intensity is plotted in the absence of a pump pulse (solid

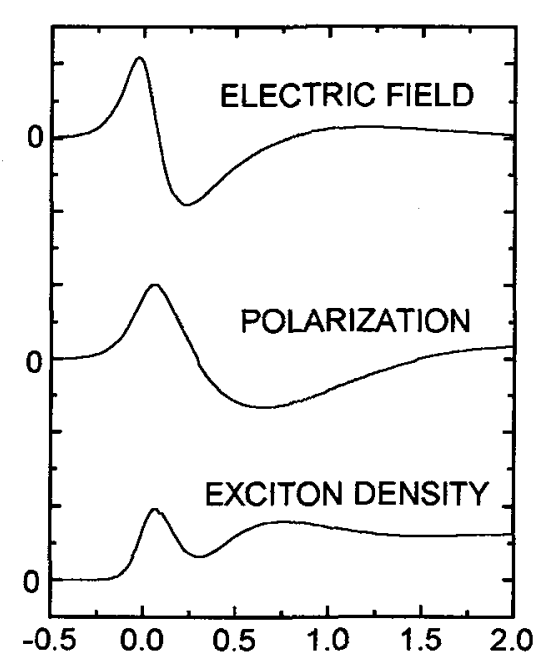


curve), and in the presence of a pump pulse 20 ps prior to the probe pulse. The change in the transmitted intensity, resulting from a reduction in the dephasing time caused by the pump pulse, is also shown in Fig. 10.

In the usual pump-and-probe experiment, one measures the change in the transmitted energy induced by a pump pulse as a function of photon energy (differential transmission spectra (DTS)). These experiments do not measure the temporal shape of the transmitted pulse. In contrast, our experiments can measure the temporal evolution of the differential transmission (DT), but do not measure the spectra. These two measurements provide complementary information. The analysis of DTS consider spectral broadening and shift of excitons, but ignore the effect of the change in the dephasing time on the transmitted pulseshape. Our DT measurements show that such effects cannot be ignored. In particular, for moderately thick sample, the net effect of a pump pulse is to decrease the transmission (i.

e. an induced absorption). This leads to the conclusion that the usual analysis of pump-and-probe experiments in terms of SBE with constant, phenomenological dephasing times must be modified. Intensity dependent dephasing rates should be introduced in SBE.

We note that these effects are likely to be less important at room temperatures because phonon interactions lead to short dephasing times and hence small distortions in the transmitted pulseshapes. Also, dephasing rates are not density dependent except at very high densities.

\section{SUMMARY}

We have discussed two new phenomena in the coherent regime for excitons: exciton-exciton interactions and pulse distortion. Exciton-exciton interaction leads to a dramatic change in the temporal shape of the four-wave-mixing signal from excitons in quantum wells. The interaction-induced nonlinear signal, which was neglected until recently, is about two-orders-of-magnitude stronger than the normal signal. We also discuss strong pulse distortion observed for low-intensity pulses propagating through moderately thick quantum well samples. The distortion increases with increasing dephasing time and a decreasing inhomogeneous linewidth. Interferometric measurements show that reradiation of the polarization induced in the quantum wells produces the pulse distortion. The pulse distortion must be considered in all linear and nonlinear coherent experiments except for very thin samples and short dephasing times.

\section{ACKNOWLEDGMENT}

It is a pleasure to acknowledge the contributions of $\mathrm{K}$. Leo, M. Wegener, S. Schmitt-Rink, W. Schaefer, E. Goebel, D. S. Chemla, T. C. Damen, J. F. Mueller, A. Vinattieri, K. Koehler, and L. N. Pfeiffer to different aspects of this work. 


\section{REFERENCES}

[1] L. Schultheis, J. Kuhl, A. Honold, and C. W. Tu, Phys. Rev. Lett. 57, 1635 (1986), 57, 1797 (1986).

[2] J. Kuhl, L. Schultheis, and A. Honold, Adv. Sollid State Phys. 29, 157 (1990).

[3] E. O. Goebel, K. Leo, T. C. Damen, J. Shah, s. Schmitt-Rink, W. Schaefer, J. F. Mueller, and K. Koehler, Phys. Rev. Lett. 64, 1801 (1990).

[4] K. Leo, J. Shah, E. O. Goebel, T. C. Damen, K. Koehler and P. Ganser, Appl. Phys. Lett. 56, 2031 (1990).

[5] B. F. Feuerbacher, J. Kuhl. R. Eccleston, and K. Ploog, Sol. St. commun. 74, 1279 (1990).

[6] K. Leo, Jagdeep Shah, E. G. Goebel, T. C. Damen,. Schmitt-Rink, W. Schaefer and K. Koehler, Phys. Rev. Lett. 66, 201 (1991).

[7] J. Feldmann, K. Leo, J. Shah, D. A. B. Miller, J. E. Cunningham, S. Schmitt-Rink, T. Meier, G. von Plessen, A. Schulze, and P. Thomas, Phys. Rev. B (1992). K. Leo, P. H. Boliviar, F. Brueggemann, R. Schwedler, Sol. St. Commun. 84, 943 (1992); P. H. Bolivar, K. Leo, J. Shah And K. Koehler, presented at the Topical Meeting on Ultrafast Electronics and Optoelectronics, San Francisco (1992).

[8] H. Roskos, M. C. Nuss, Jagdeep Shah, K. Leo, D. A. B. Miller, A. M. Fox, K. Koehler and P. Ganser, Phys. Rev. Lett. 68, 2216 (1992).

[9] K. Leo, M. Wegener, J. Shah, D. S. Chemla, E. O. Göbel, T. C. Damen, S. Schmitt-Rink, W. Schäfer, Phys. Rev. Lett. 65, 1340 (1990).

[10] M. Wegener, D. S. Chemla, S. Schmitt-Rink, and W. Schäfer, Phys. Rev. A 42, 5675 (1990),

[11] D. S. Kim, J. Shah, T. C. Damen, W. Schäfer, F. Jahnke, and S. Schmitt-Rink, Phy. Rev. Lett. 69, 2725 (1992).

[12] H. Haug and S. W. Koch, "Quantum Theory of the Optical and Electronic Properties of Semiconductors", World Scientific, Singapore, 1990; Ch. 12.

[13] T. Yajima and Y. Taira, J. Phys. Soc. Jpn. 47, 1620 (1979).

[14] H. -J. Hartmann and A. Laubereau, J. Chem. Phys. 80, 4663 (1984).

[15] D. Fröhlich, A. Kulik, B. Uebbing, A. Mysyrowica, V. Langer, H. Stolz, and W. von der Osten, Phys. Rev. Lett. 67, 2343 (1991).

[16] D. S. Kim, Jagdeep Shah, A. Vinattieri, T. C. Damen, D. A. B. Miller, QPD18, postdeadline paper presented at QELS'93; also, Kim et al., submitted to PRL.

[17] F. Tassone, F. Bassani, and L. C. Andreani, Il Nuovo Cimento 12 D, 1673 (1990).

[18] Kim et al., unpublished. 